\title{
Tetraphenylporphyrin Enters the Ring: First Example of a Complex Between Highly Bulky Porphyrins and a Protein
}

\author{
Yuma Shisaka ${ }^{1}$, Erika Sakakibara ${ }^{1}$, Kazuto Suzuki ${ }^{1}, J^{\prime}$ shua Kyle Stanfield ${ }^{1}$, Hiroki Onoda ${ }^{1}$, \\ Garyo Ueda ${ }^{1}$, Miu Hatano ${ }^{1}$, Hiroshi Sugimoto ${ }^{2,3}$, and Osami Shoji ${ }^{1,3^{*}}$ \\ ${ }^{1}$ Department of Chemistry, Graduate School of Science, Nagoya University, Furo-cho, Chikusa-ku, \\ Nagoya 464-8602, Japan. \\ ${ }^{2}$ RIKEN SPring-8 Centre, 1-1-1 Kouto, Sayo, Hyogo 679-5148, Japan. \\ ${ }^{3}$ Core Research for Evolutional Science and Technology, Japan Science and Technology Agency, 5 \\ Sanbancho, Chiyoda-ku, Tokyo, 102-0075, Japan.
}

*To whom correspondence should be addressed.
E-mail: shoji.osami@a.mbox.nagoya-u.ac.jp

\begin{abstract}
Tetraphenylporphyrin (TPP) is a synthetic porphyrin whose properties can be readily modified, endowing it with significant benefits over naturally occurring porphyrins. Yet, their insolubility in water and/or steric bulk have rendered them incompatible with biological systems. Herein, we report the first example of a native biomolecule capturing TPP as well as its derivatives. The haemoprotein HasA, secreted by certain pathogens to scavenge haem from their hosts, can capture various metal- and mesosubstituted TPPs. The rapid crystallisation of TPP derivatives captured by HasA revealed the binding mode of TPP at excellent resolutions. A single-site mutation (L85A) of HasA enlarged the binding pocket, allowing the incorporation of a bulkier derivative of TPP. HasA binding TPP derivatives was also demonstrated to inhibit proliferation of the opportunistic pathogen Pseudomonas aeruginosa. This study not only represents a simple method for the complexation of TPP derivatives with a native protein, but also opens the door for the future use of TPP derivatives as biological tools.
\end{abstract}




\section{Main text}

Porphyrins, such as haem and chlorophyll, are ubiquitous in nature, where they are generally found associated with proteins. Haemoproteins represent a major class of metalloproteins and are responsible for a plethora of essential biological functions. Owing to their biological significance, countless synthetic porphyrins have been developed as models of haemoproteins. ${ }^{1-3}$ Amongst synthetic porphyrins, tetraphenylporphyrin (TPP) represents the most common and historically significant one, possessing a structure that is not only simpler but also of a higher symmetry than naturally occurring $\beta$ substituted porphyrins. The first synthetic route for the preparation of TPP was reported by Lord Rothemund in 1935, ${ }^{4,5}$ and subsequently improved by A. D. Adler and F. R. Longo in $1966 .{ }^{6,7}$ The establishment of facile synthetic routes for the insertion of four distinct meso substituents by J. S. Lindsey further propelled the research of TPP and its meso-substituted derivatives into a new phase. ${ }^{8-10}$ These impressive developments have culminated in the design of myriad TPP derivatives possessing diverse catalytic properties ${ }^{11-16}$ as well as applications as photosensitisers for photodynamic therapy ${ }^{17-}$ ${ }^{19}$ and building blocks in porphyrin-based architectures. ${ }^{20-25}$

It is well established that the nature of haemoproteins is intimately linked to the physicochemical properties of the haem cofactor. Consequently, the reconstitution of haemoproteins with synthetic metalloporphyrins has been avidly studied as a promising means to alter their properties. ${ }^{26-30}$ Unfortunately, the high selectivity of native haemoproteins for haem has imposed crippling restrictions upon the structural diversity of metalloporphryins that can be employed. For example, although the incorporation of TPP derivatives into proteins is highly desired, owing to the optical and redox properties of TPP being readily influenced by the meso substituent, this has been impossible, due to the high steric bulk of TPP, which is further exacerbated by its extreme hydrophobicity. In the case of de novo proteins, some meso-substituted synthetic porphyrins are accepted; ${ }^{31-33}$ however, accommodation of TPP has yet to be achieved. Recently, we reported that the haemoprotein HasA, which is secreted by certain pathogens, such as Pseudomonas aeruginosa, to pirate haem from its host (Fig. 1a), can accept various bulky synthetic metalloporphyrins, such as diphenylporphyrin. ${ }^{34-37}$ Yet, at this time, we were unable to incorporate bulkier TPP into HasA and concluded that this may not be possible, ${ }^{34,35}$ and that the TPP skeleton may be incompatible for incorporation into unadulterated proteins and application with natural biological systems.

Herein we overturn this notion by reporting the first successful and stable incorporation of a series of metalloTPPs into a native haemoprotein, HasA, made possible by the facile optimisation of the buffer conditions during purification (Fig. 1b). Moreover, a single amino acid mutation of HasA is sufficient to enlarge the cavity, enabling its use as a vessel for even bulkier derivatives of TPP. Furthermore, we investigated whether TPP derivatives captured by HasA can inhibit the proliferation of P. aeruginosa, an opportunistic bacterium that has been categorised as a "critical priority pathogen" by the WHO in $2017 . .^{38}$ 
a
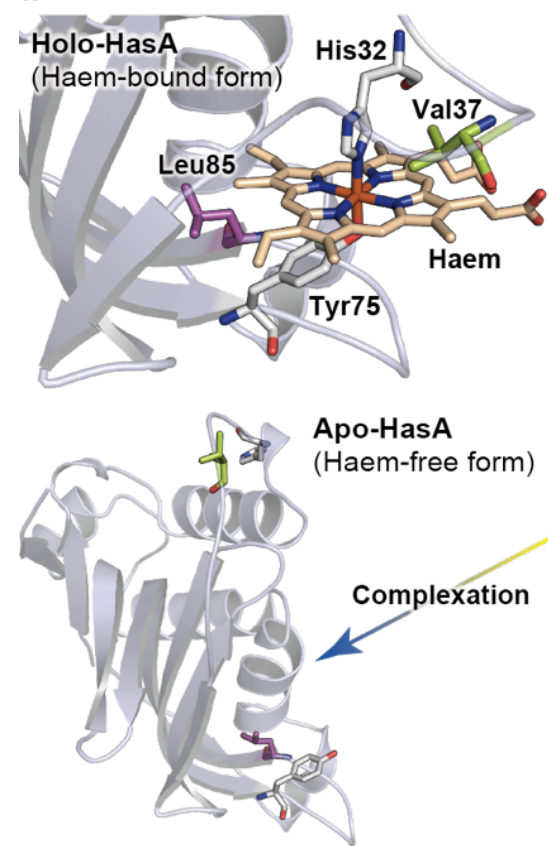

b

Tetraphenylporphyrin (TPP)

derivatives (this work)

1. Metal-substituents

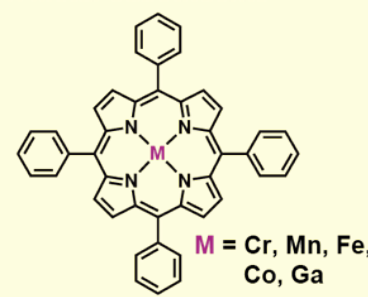

2. Meso-substituents

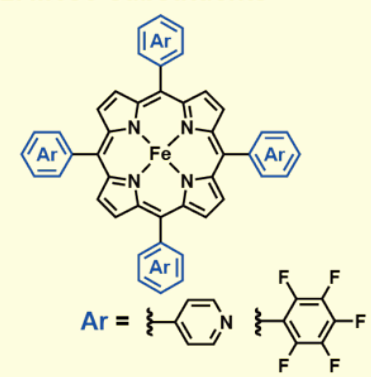

Figure 1 | Concept of this study. (a) Haem-acquisition protein HasA from Pseudomonas aeruginosa. Crystal structures of the haem-bound form (holo-HasA, PDB ID: 3ELL) and haem-free form (apo-HasA, PDB ID: 3MOK) manifest significantly different haem-binding site architectures. (b) Structures of the TPP derivatives employed in this study.

\section{Results}

\section{Characterisation and X-ray crystal structure analysis of HasA in complex with metalloTPPs.}

As touched upon in the introduction, based upon our previous findings, we concluded that the iron complex of TPP (FeTPP) was too bulky for incorporation into HasA. Through careful observation, however, we found that FeTPP actually does bind to HasA, but the resulting complex is unstable in Tris buffer at pH 7.0, and FeTPP gradually dissociates from HasA over time (Supplementary Fig. 1a). Moreover, by exchanging the buffer solution with ammonium sulphate, a broadening of the Soret band could be observed, indicating a change in the coordination of FeTPP (Supplementary Fig. 1b-c). This suggests that the composition and/or $\mathrm{pH}$ of the buffer may be the key to obtaining a stable FeTPP-HasA complex. Thus, we set out to examine if a stable complex of FeTPP-HasA can be obtained by modifying the buffer conditions. Unlike in routinely employed neutral buffers, e.g. Tris and phosphate, notable stabilisation of the complex between HasA and FeTPP (FeTPP-HasA) could be attained in the alkaline buffer CHES. The resulting FeTPP-HasA complex was stable even after purification, and the ESI-TOF mass spectrum showed a clear peak corresponding to the mass of FeTPP-HasA, confirming the successful formation of a stable complex (Fig. 2a, b). Analysis of the FeTPP-HasA complex by UVVis spectroscopy showed characteristic absorption between 300-750 nm derived from FeTPP (Supplementary Fig. 1d).

To determine the correct incorporation of FeTPP in the protein's haem-binding site, purified FeTPPHasA was crystallised, and its structure was determined up to a resolution of $1.5 \AA$ by X-ray diffraction (Fig. 2c-e and Supplementary Table 1). Similar to the native haem cofactor, FeTPP is coordinated by 
His32 and Tyr75 of HasA (Fig. 2c). The overall fold of HasA remained largely unperturbed, with the overall $\alpha$-carbon root-mean-square deviation (C $\alpha$-RMSD) between FeTPP-HasA and haem-HasA (holo-HasA) being 0.72 . The most notable difference was in the orientation of the loop that forms the haem-binding pocket of HasA (Fig. 2d). Owing to loop's flexible nature, accommodation of bulky FeTPP with its four phenyl groups became possible through a small $0.7 \AA$ shift of the centre of FeTPP towards the outside of HasA. Interestingly, HasA can also capture and form stable complexes with TPP harbouring other metals, such as the d-block transition metals chromium, manganese, and cobalt as well as the p-block metal gallium (Supplementary Figs. 2-5). Comparison of X-ray crystal structures of HasA-bound metalloTPPs ( $\mathrm{Cr}, \mathrm{Mn}, \mathrm{Co}$ ) revealed that, whilst their thermal stabilities differ markedly from FeTPP $(\mathrm{Cr}>\mathrm{Co}>\mathrm{Mn}>\mathrm{Fe}$ ) (Supplementary Fig. 3), their mode of binding is the same as for FeTPP (Supplementary Fig. 4 and Supplementary Tables 1-2). In the case of GaTPP-HasA, incorporation of GaTPP was confirmed by ESI-TOF MS and X-ray absorption near-edge structure (XANES) spectroscopy (Supplementary Fig. 5). Of note, clear electron densities corresponding to constituents of the final buffer solution for crystallisation (CHES and CAPS), were observed with high occupancies at the interface between two HasA monomers (Supplementary Fig. 6), indicating that these buffering agents may play an important role in the stabilisation of HasA-bound metalloTPPs as well as their rapid crystallisation, which completed within 3 hours.

a

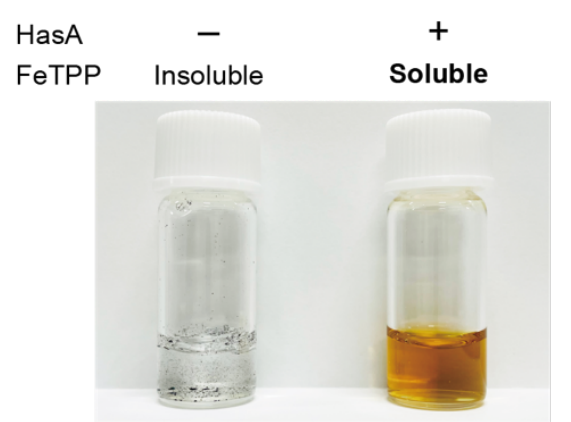

b

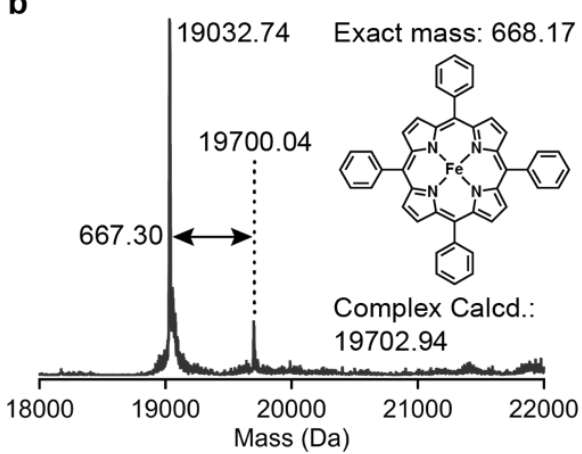

C

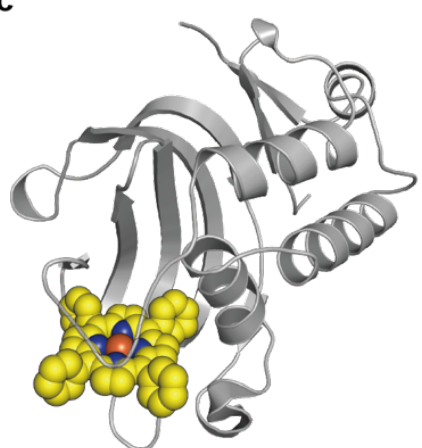

d

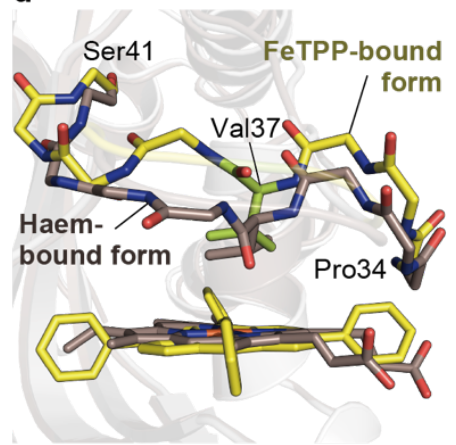

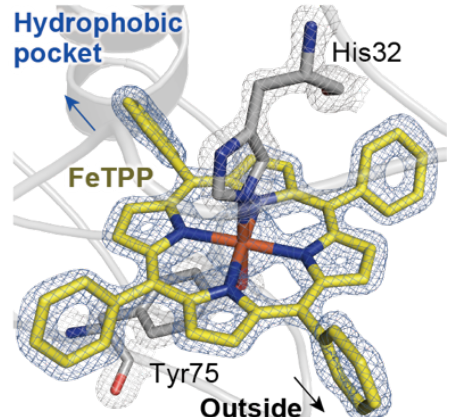

Outside

e

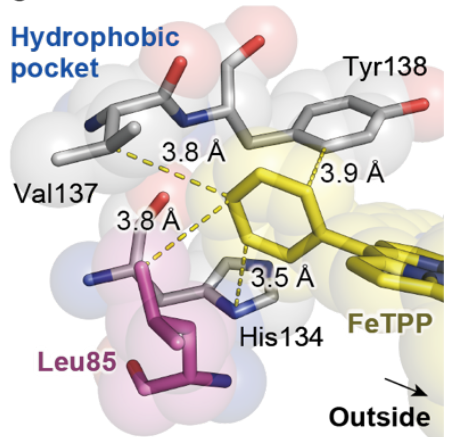

Figure 2 | Characterisation and crystal structure analysis of FeTPP-HasA. (a) Solution of FeTPP in $0.1 \mathrm{M}$ CHES-KOH (pH 9.5) in the presence (right) and absence (left) of HasA. (b) Positive ion mode ESI-TOF mass spectrum of FeTPP-HasA in $20 \mathrm{~mm}$ ammonium acetate. Molecular weight of complex-free HasA (apo-HasA) was calculated to be 19034.77 Da. (c) Crystal structure of the complex between FeTPP and HasA (left). FeTPPbinding site of HasA (right). $2 F_{\mathrm{o}}-F_{\mathrm{c}}$ map contoured at $2.5 \sigma$ (grey mesh). $F_{\mathrm{o}}-F_{\mathrm{c}}$ map omitting FeTPP contoured at $5.0 \sigma$ (blue mesh). (d) Flexible loop coding His32 ligand shows large conformational change compared with HoloHasA (brown). Only the main chain atoms are rendered. Side chain also shown for Val37. (e) Hydrophobic pocket of HasA incorporates one phenyl group of FeTPP. 


\section{Meso-substituents of TPP in complex with HasA and its mutant.}

The successful incorporation of TPP into HasA has opened up the door to a host of new possibilities. For example, iron tetra(4-pyridyl)porphyrin (FeTPyP) has already been utilised as a scaffold in the formation of supramolecular architectures, which are expected to find applications as chemical and biomedical tools. We found that HasA can also accommodate FeTPyP, employing the same reconstitution method used for metalloTPPs (Supplementary Fig. 7). Furthermore, crystal structure analysis of this complex revealed that two 4-pyridyl groups are solvent exposed, extending towards the outside of Has A (Fig. 3a). This puts them in an auspicious position for the future formation of protein assemblies. On the other hand, a unique bulkier derivative of FeTPP, namely iron mesotetrakis(pentafluorophenyl)porphyrin (FeTPFPP), which was expected to confer HasA with interesting catalytic properties, could not be accommodated by wild-type HasA, on account of the increased $\mathrm{C}-\mathrm{F}$ bond length and van der Waals radius of fluorine. ${ }^{39}$ However, FeTPFPP was able to fit into the haembinding site through mutation of Leu85, nestled in the hydrophobic pocket of HasA close to one of the phenyl groups of FeTPP (Fig. 2e), to less bulky alanine (L85A) (Fig. 3b, c). The complex between FeTPFPP and HasA(L85A) was maintained even after anion-exchange chromatography (Supplementary Fig. 8a). ESI-TOF mass spectrometry further substantiated the stable binding of FeTPFPP to HasA(L85A) (Fig. 3c). Whilst we could not obtain crystals of FeTPFPP-HasA(L85A), the crystal structure of FeTPP-HasA(L85A) suggested that removal of the isopropyl moiety from Leu85 allows the isopropyl side chain of Val137 to flip into this space, thereby generating sufficient room to accommodate a pentafluorophenyl group of FeTPFPP (Fig. 3d). 

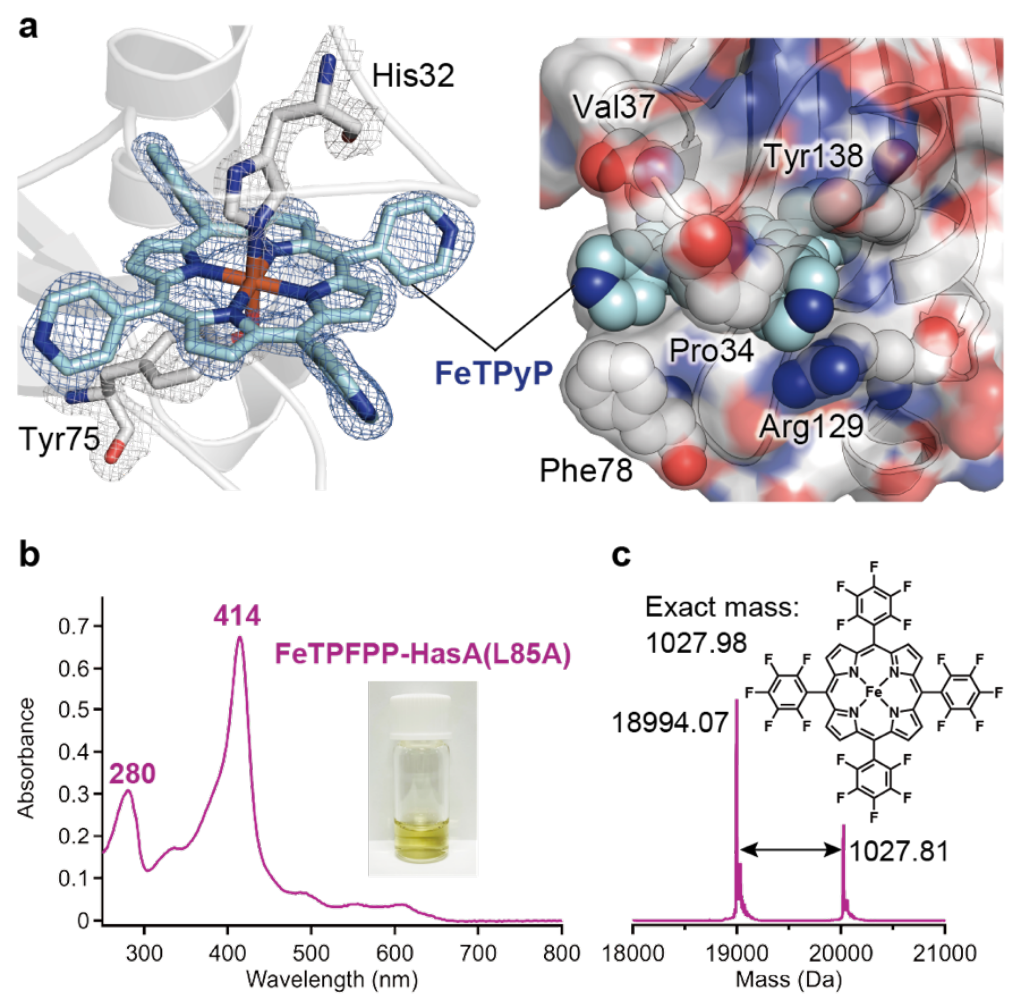

d

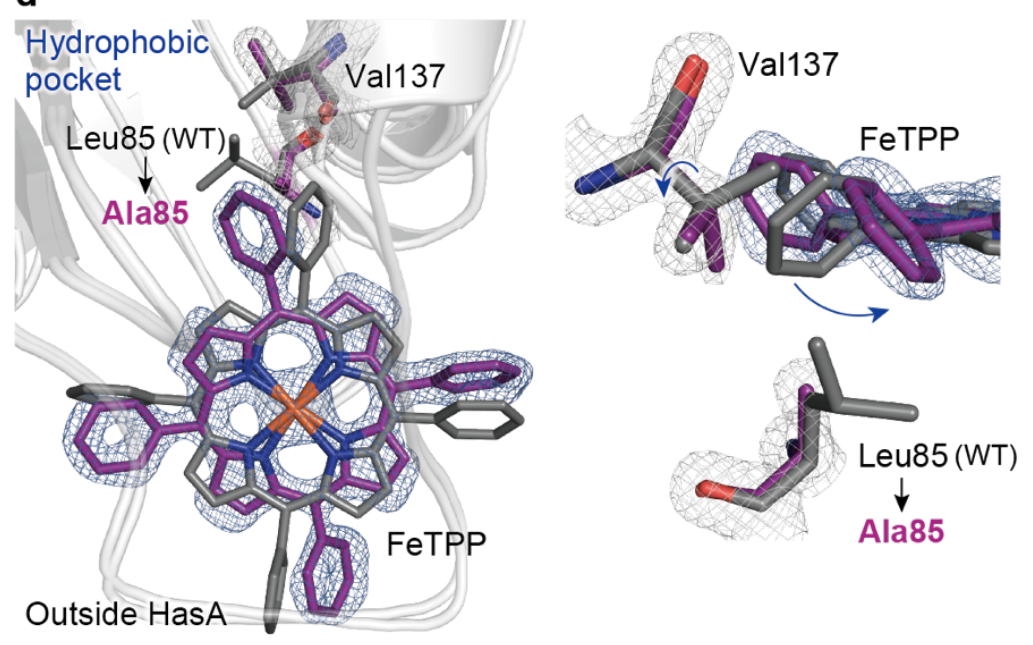

Figure 3 | Meso-substituents of TPP captured by HasA. (a) Crystal structure of FeTPyP-HasA. $2 F_{0}-F_{c}$ map contoured at $2.0 \sigma$ (grey mesh). $F_{\mathrm{o}}-F_{\mathrm{c}}$ map omitting FeTPyP contoured at $3.5 \sigma$ (blue mesh). (b) UV-Vis spectrum and solution of FeTPFPP-HasA(L85A). (c) Positive-mode ESI-TOF mass spectrum of FeTPFPP-HasA(L85A) in $20 \mathrm{~mm}$ ammonium acetate. Molecular weight of HasA(L85A) was calculated to be $18992.69 \mathrm{Da}$. (d) Superposed image of the crystal structures of the wild type (WT, grey) and L85A mutant (purple) of HasA capturing FeTPP. Enlarged view of mutated Leu85 and neighbouring Val137 is shown on the right. $2 F_{\mathrm{o}}-F_{\mathrm{c}}$ map contoured at $2.0 \sigma$ (grey mesh). $F_{\mathrm{o}}-F_{\mathrm{c}}$ omitting FeTPP contoured at $4.0 \sigma$ (blue mesh). 


\section{Inactivation of $P$. aeruginosa utilising TPP derivatives captured by HasA.}

We evaluated the potential of TPP derivatives captured by HasA to be used as bacteriostats of $P$. aeruginosa. Our research group has previously reported that HasA capturing iron phthalocyanine and iron 5,15-diazaporphyrin inhibit the proliferation of $P$. aeruginosa under iron-limiting condition..$^{34,35}$ Initial investigation was conducted on a standard strain of $P$. aeruginosa called PAO1. In iron-limiting growth medium, P. aeruginosa PAO1 grew through acquisition of haem from holo-HasA that had been supplemented to the medium as the sole source of iron. Subsequently, the bacteriostatic effect of HasA capturing TPP derivatives on the proliferation of PAO1 in the presence of holo-HasA was monitored. Interestingly, all tested metalloTPP-HasAs could inhibit the growth of PAO1 to varying degrees (Fig. 4a). In particular, CoTPP-HasA was found to be an extremely efficient inhibitor of PAO1 growth. More importantly, we could also demonstrated that CoTPP-HasA is effective against multiple strains of multidrug-resistant $P$. aeruginosa (MDRP), which are intractable using current antibiotics (Fig. $4 \mathrm{~b}-\mathrm{d}$ ). ${ }^{40}$ Given that there are no structural perturbations observed between the crystal structures of HasA capturing either haem or TPP derivatives, HasA incorporating metalloTPPs is expected to interact with the HasA-receptor HasR on the outer membrane of P. aeruginosa in a similar fashion as holo-HasA. From measurements of the thermal stability of metalloTPP-HasA complexes, we know that there exists no correlation between growth inhibition and complex stability. It follows that the TPP derivatives are likely transferred to HasR via the formation of a HasA-HasR complex. Furthermore, the extremely strong binding of TPP derivatives, especially CoTPP, to HasR may cause continuous blocking of the HasR channel. This is thought to suppress dissociation of apo-HasA from HasR, thus preventing further rounds of haem uptake, and ultimately leading to the cessation of growth (Fig. 4g). We believe that further investigation will allow us to elucidate the detailed molecular mechanism behind this type of bacterial inactivation.

A different approach to inactivate $P$. aeruginosa was based upon the photophysical property of GaTPP, to generate singlet oxygen following photoirradiation, in combination with HasA as a Trojan horse to smuggle GaTPP into the bacterial cell. The bactericidal potential of GaTPP-HasA was examined as follows; P. aeruginosa was pre-incubated with $1 \mu \mathrm{M}$ GaTPP-HasA and subsequently irradiated with light at $420 \mathrm{~nm}$, which corresponds to the Soret band of GaTPP-HasA. Photosterilisation of $P$. aeruginosa PAO1 was successful with the efficiency exceeding 99.99\% (Fig. 4e). Notably, GaTPP-HasA also sterilised MDRPs with a comparable efficiency to PAO1 (Fig. 4e). More detailed investigation by fluorescence microscopy revealed that, in contrast to wild-type PAO1, fluorescence from GaTPP cannot be observed when the hasR knockout mutant ( $\triangle$ has $R$ ) is incubated with GaTPPHasA (Fig. 4f). This indicates that GaTPP-HasA specifically binds to the HasA-receptor HasR on the outer membrane of $P$. aeruginosa, and GaTPP captured by HasA is taken up into $P$. aeruginosa cells through HasR (Fig. 4g). Photoirradiation thus results in the generation of singlet oxygen inside of $P$. aeruginosa, inducing bacterial cell death. 

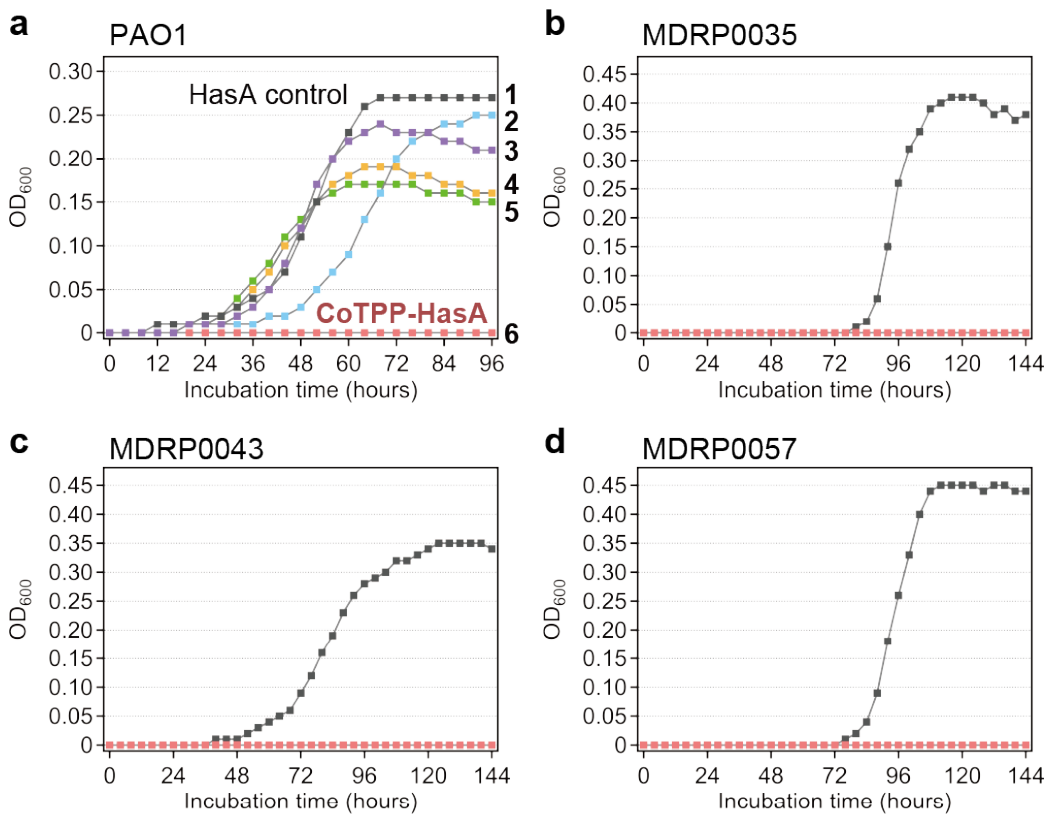

- HasA control (1) - FeTPFPP-HasA(L85A) (2) - MnTPP-HasA (3)

- FeTPP-HasA (4) $=$ CrTPP-HasA (5) $=$ CoTPP-HasA (6)
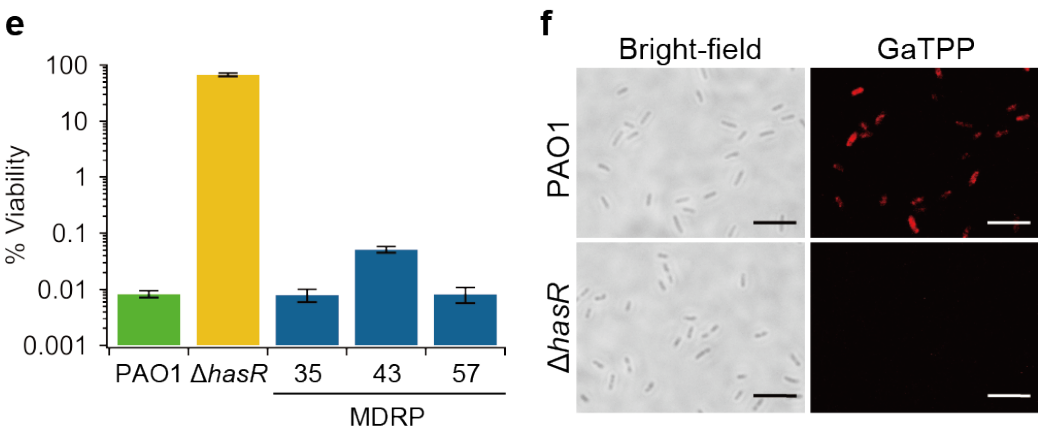

\section{g Growth inhibitior}
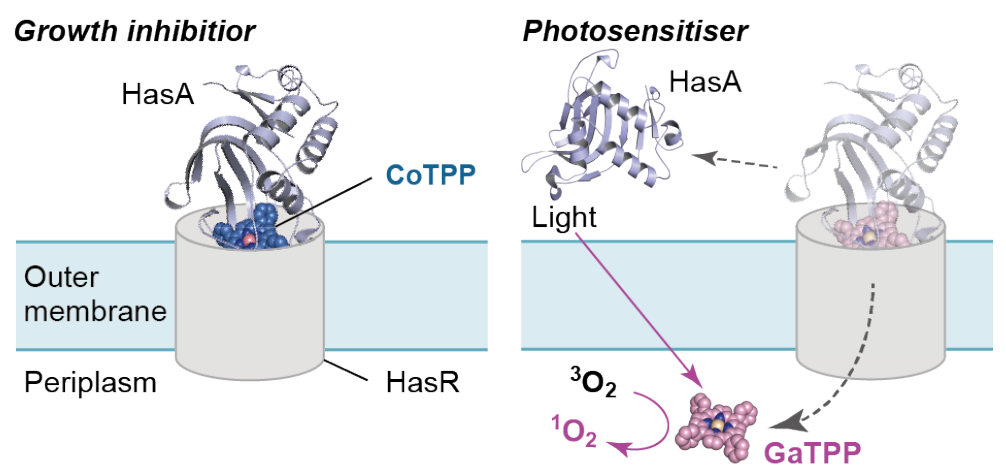

Figure 4 Growth inhibition and photosterilisation of $P$. aeruginosa utilising HasA capturing TPP derivatives in iron-limiting medium. (a-d) Growth curves of $P$. aeruginosa. All conditions contain $1 \mu \mathrm{M}$ holoHasA as the sole iron source. $1 \mu \mathrm{M}$ HasA capturing TPP derivatives was also added except for HasA control. Bacterial strain is shown on each graph. (e) Photosterilisation of $P$. aeruginosa treated with $1 \mu \mathrm{M}$ GaTPP-HasA. Data is shown as the mean $\pm \mathrm{sd}(n=3)$. (f) Microscope images of $P$. aeruginosa PAOl and $\Delta$ has $R$ incubated with GaTPP-HasA. Scale bars, $6 \mu \mathrm{m}$. (g) Proposed mechanisms of growth inhibition and photosterilisation utilising HasA capturing TPP derivatives. 


\section{Discussion}

In conclusion, we have confirmed the first successful example of a complex between TPP and a protein. Our structural study also reveals a propensity of HasA to capture various metalloTPPs. Execution of site-directed mutagenesis to enlarge the hydrophobic pocket of HasA enabled the construction of mutants capable of capturing a bulkier fluorinated TPP derivative. This demonstrates that rearrangement of the hydrophobic pocket may potentially permit the complexation of Has A with a wide variety of meso-substituted porphyrins. Our work further supports a potential application for TPP derivatives in the inactivation of multidrug-resistant strains of $P$. aeruginosa (MDRP). Owing to the hydrophobicity and steric bulk of TPP being overcome by complexation with HasA, we anticipate a wealth of new uses to emerge for TPP derivatives in biological systems.

Whilst this report is restricted to the construction of HasA capturing five metalloTPPs and two mesosubstituted TPP derivatives, followed by the inactivation of MDRP, we believe these findings may usher in a new chapter in porphyrin chemistry, wherein TPP and its derivatives can finally be applied in the field of microbiology, e.g. as porphyrin-based antimicrobials, and in protein engineering, e.g. in the construction of artificial enzymes and assembly of supramolecular architectures. Investigations in this direction are currently underway in our research group.

\section{Methods}

Synthesis of TPP derivatives. The syntheses of $\mathrm{FeTPPCl}^{7}$ tetra(4-pyridyl)porphyrin iron chloride $(\mathrm{FeTPyPCl}){ }^{41}$ and meso-tetrakis(pentafluorophenyl)porphyrin iron chloride (Fe(TPFPP)Cl) ${ }^{42,43}$ were conducted according to previous reports. HRMS (ESI) Calcd. for $\mathrm{C}_{44} \mathrm{H}_{28} \mathrm{ClFeN}_{4}$ [M]: 703.1352. Found: 703.1324; Calcd. for $\mathrm{C}_{40} \mathrm{H}_{25} \mathrm{ClFeN}_{8}[\mathrm{M}+\mathrm{H}]$ : 708.1240. Found: 708.1242; Calcd. for $\mathrm{C}_{44} \mathrm{H}_{7} \mathrm{ClF}_{20} \mathrm{FeN}_{4}$ [M-H]: 1061.9389. Found: 1061.9388. Other metal derivatives of TPP (Metal $=\mathrm{Cr}, \mathrm{Mn}, \mathrm{Co}, \mathrm{Ga})$ were purchased from Porphyrin-Laboratories $\mathrm{GmbH}$.

Plasmid construction. Expression plasmid for L85A mutant of HasA was constructed by using the plasmid pQE30t containing the hasAp(T) gene ${ }^{34}$ as the template for polymerase chain reaction (PCR). Sequences of primers are as follows; Fwd: 5'-ACCGCGTGGGGCAAGCTGGACAG-3', and Rev: 5'GTGGCTGGGGTTGCTGAACAGG-3'. Resulting plasmid was introduced into Escherichia coli strain M15 [pREP4] to overexpress HasA(L85A) according to the same method as wild-type HasA reported previously. ${ }^{34}$

Protein expression and purification. Purified haem-free HasA (apo-HasA) was prepared according to a study by Shirataki et al. ${ }^{34} \mathrm{E}$. coli cells expressing HasA were stored at $-80{ }^{\circ} \mathrm{C}$ until further use. The general procedure to construct HasA capturing metalloTPPs was as follows (FeTPP-HasA as an example). Synthesised tetraphenylporphyrin iron(III) chloride complex was solubilised in DMSO, and then mixed with apo-HasA in PBS. Following overnight dialysis of a mixture of FeTPP/HasA in $50 \mathrm{mM}$ Tris- $\mathrm{HCl}(\mathrm{pH} 8.0)$, excess FeTPP was removed by filtration through a PVDF membrane $(0.22 \mu \mathrm{m}$, 
Millipore). The filtrate was loaded onto a Q Sepharose Fast Flow column (GE Healthcare) preequilibrated in $50 \mathrm{mM}$ Tris- $\mathrm{HCl}(\mathrm{pH}$ 8.0) buffer and washed with at least 3 column volumes of buffer A [0.1 M CHES-KOH (pH 9.5)]. Bound protein was eluted in buffer A containing $0.5 \mathrm{M} \mathrm{NaCl}$. After desalting, the eluates were purified via anion exchange chromatography (HiPrep capto DEAE $5 \mathrm{~mL}$; GE Healthcare) to eliminate apo-HasA. The loaded sample was washed with 1 column volume of buffer A including $10 \%$ buffer $\mathrm{B}$ [buffer A with $0.8 \mathrm{M} \mathrm{NaCl}$ ], and then gradually eluted over 20 column volumes with the ratio of buffer B increasing gradually from $10 \%$ to $80 \%$. Purified FeTPP-HasA was stored in $0.1 \mathrm{M}$ CHES-KOH $\left(\mathrm{pH} 9.5\right.$ ) at $4{ }^{\circ} \mathrm{C}$ or $-80{ }^{\circ} \mathrm{C}$. The final protein concentration was estimated via a Pierce ${ }^{\mathrm{TM}}$ BCA Protein Assay Kit (Thermo Scientific). Apo-HasA $\left(\varepsilon_{280}=28.6 \mathrm{mM}^{-1} \mathrm{~cm}^{-1}\right)^{44}$ was used as the protein standard. The purified derivatives of TPP-HasA were analysed by UV-Vis spectroscopy and ESI-TOF MS (see Supplementary Figures). The estimated molar extinction coefficients of HasA capturing TPP derivatives are as follows: FeTPP-HasA: $\varepsilon_{428}=91.9 \mathrm{~mm}^{-1} \mathrm{~cm}^{-1}$; CrTPP-HasA: $\quad \varepsilon_{453.5}=234.8 \mathrm{~mm}^{-1} \mathrm{~cm}^{-1} ; \quad$ MnTPP-HasA: $\quad \varepsilon_{463}=92.3 \mathrm{~mm}^{-1} \mathrm{~cm}^{-1} ; \quad$ CoTPP-HasA: $\varepsilon_{437}=221.3 \mathrm{~mm}^{-1} \mathrm{~cm}^{-1}$; GaTPP-HasA: $\varepsilon_{434}=298.8 \mathrm{mM}^{-1} \mathrm{~cm}^{-1}$; FeTPyP-HasA: $\varepsilon_{428}=82.9 \mathrm{~mm}^{-1} \mathrm{~cm}^{-1}$; FeTPFPP-HasA(L85A): $\varepsilon_{414}=63.8 \mathrm{mM}^{-1} \mathrm{~cm}^{-1}$; FeTPP-HasA(L85A): $\varepsilon_{430}=85.7 \mathrm{mM}^{-1} \mathrm{~cm}^{-1}$.

Crystallisation of HasA capturing TPP derivatives. For crystallisation, purified protein samples were exchanged into 0.1 M CHES-KOH (pH 9.5) buffer via PD-10 desalting columns (GE Healthcare). The eluates were concentrated in an Amicon Ultra filter tube with a $3 \mathrm{kDa}$ MWCO (Merck Millipore) and the concentration of protein samples was determined by UV-Vis spectroscopy. Screening of crystallisation conditions was carried out with Wizard ${ }^{\mathrm{TM}}$ Classic 1 and 2 (Rigaku). Equivalent volumes of protein sample and reservoir solution were mixed and incubated at $20^{\circ} \mathrm{C}$ by sitting-drop vapourdiffusion. Unless otherwise noted, crystals were flash-cooled in a nitrogen cryostream at $100 \mathrm{~K}$ after soaking in a suitable reservoir solution containing $30-40 \%$ glycerol. Final conditions for crystallisation were as follows.

MetalloTPP-HasAs: A 1.0 $\mu \mathrm{L}$ aliquot of wild-type HasA capturing metalloTPPs (Metal $=\mathrm{Cr}, \mathrm{Mn}, \mathrm{Fe}$, Co) [1.5 mM in 0.1 M CHES-KOH buffer ( $\mathrm{pH} 9.5$ )] was mixed with an equal volume of reservoir solution [0.1 M CAPS-NaOH (pH 10.5), $1.2 \mathrm{M} \mathrm{NaH}_{2} \mathrm{PO}_{4}, 0.8 \mathrm{M} \mathrm{K}_{2} \mathrm{HPO}_{4}$, and $0.2 \mathrm{M} \mathrm{Li}_{2} \mathrm{SO}_{4}$ ].

FeTPyP-HasA: A $1.0 \mu \mathrm{L}$ aliquot of HasA capturing FeTPyP [4.6 mM in $0.1 \mathrm{M}$ CHES-KOH buffer

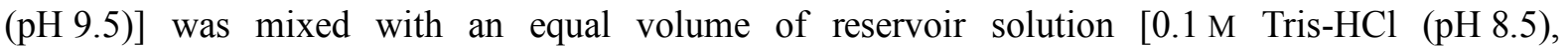
$1.26 \mathrm{M}\left(\mathrm{NH}_{4}\right)_{2} \mathrm{SO}_{4}$, and $\left.0.2 \mathrm{M} \mathrm{Li}_{2} \mathrm{SO}_{4}\right]$. Crystals of FeTPyP-HasA were cryoprotected by soaking in reservoir solution containing $30 \%$ glycerol and then flash-frozen and stored in liquid nitrogen until Xray diffraction experiments.

FeTPP-HasA(L85A): A $1.0 \mu \mathrm{L}$ aliquot of the HasA L85A mutant capturing FeTPP [1.5 mM in $0.1 \mathrm{M}$ CHES-KOH buffer ( $\mathrm{pH}$ 9.5)] was mixed with an equal volume of reservoir solution [0.1 M Tris-HCl (pH 8.5), 20\% PEG 8000, and $0.2 \mathrm{M} \mathrm{MgCl}_{2}$ ].

Data collection and structure refinement. X-ray diffraction data sets were collected at 
RIKEN/SPring-8 centre (Hyogo, Japan) on beamlines BL26B1 equipped with either an MX225-HE CCD or EIGER X 4M detector, BL26B2 equipped with an MX225-HS CCD detector, and BL41XU equipped with an EIGER X 16M detector with a wavelength of $1.000 \AA$ at $100 \mathrm{~K}$. Anomalous datasets and X-ray fluorescence scan data were also collected from the same crystal used during single data collection (Supplementary Table 2). Reflection data was automatically processed with $X D S^{45}$ implemented in the $K A M O$ software ${ }^{46}$ or integrated by HKL2000, ${ }^{47}$ followed by scaling with Aimless or XSCALE. ${ }^{48-50}$ Initial phases were determined by molecular replacement method using MOLREP. ${ }^{51}$ The structure of haem-HasA (Holo-HasA, PDB ID: 3ELL) ${ }^{52}$ was used as the search model for molecule replacement. Model building and refinement were performed on $C o o t^{53}$ and REFMAC5 ${ }^{54-56}$ The models of TPP derivatives were generated using $P R O D R G^{57}$ and $e L B O W^{58}$ for the refinement process. All protein figures were drawn on PyMOL2. The final refinement statistics are summarised in Supplementary Table 1.

Circular Dichroism (CD) Spectroscopy. Thermal stability of HasA capturing metalloTPPs was analysed by CD spectroscopy (J-720WN (JASCO)). Molar ellipticity at $222 \mathrm{~nm}$ was measured as a function of temperature from $35{ }^{\circ} \mathrm{C}$ to $95{ }^{\circ} \mathrm{C}$ at increments of $1.0{ }^{\circ} \mathrm{C}$ per minutes controlled by a Peltier thermostat. Temperatures upon thermal denaturation $\left(T_{\mathrm{m}}\right)$ were determined by a previously reported method. ${ }^{59}$

X-ray absorption near-edge structure (XANES) analysis. XANES spectra were collected on the BL36XU beamline at SPring-8 using an Si ( $\left.\begin{array}{lll}1 & 1 & 1\end{array}\right)$ channel cut monochromator (CCM). Energies were calibrated using $\beta-\mathrm{Ga}_{2} \mathrm{O}_{3}$ with the K-edge being assigned as $10375.1 \mathrm{eV}$. Standard samples were analysed by transmission mode quick XAFS (QXAFS). The XANES spectrum of a solution of GaTPPHasA was measured in fluorescence mode at the gallium K-edge at room temperature. XANES spectra were averaged using weights based on the signal-to-noise ratio, with each spectrum checked individually prior to averaging. Averaged spectra were normalised with the post-edge baseline being assigned an absorbance of 1 . Results were analysed on Athena. ${ }^{60}$

Strains of $\boldsymbol{P}$. aeruginosa used in this study. P. aeruginosa strain PAO1 was used as the standard strain. A previously constructed has $R$ gene knockout mutant ( $\operatorname{has} R)$ of PAO1 was used. ${ }^{37}$ Three types of multidrug-resistant strains of $P$. aeruginosa (MDRP0035, MDRP0043, and MDRP0057) ${ }^{40}$ were further investigated.

Monitoring the growth of $P$. aeruginosa in iron-limiting medium containing HasA capturing TPP derivatives. Growth experiments of $P$. aeruginosa were performed according to a previously reported method. ${ }^{35} 1 \mu \mathrm{M}$ holo-HasA was added as the sole iron source to all cultures. To evaluate the efficiency of growth inhibition of HasA capturing TPP derivatives, these artificial-HasA were also added to a final concentration of $1 \mu \mathrm{M}$. Holo-HasA was prepared according to a report by Shirataki et al. ${ }^{34}$ 
Photosterilisation of $\boldsymbol{P}$. aeruginosa utilising GaTPP-HasA. Photosterilisation of $P$. aeruginosa was performed according to previously reported conditions, ${ }^{37}$ with the exception of the wavelength used for photoirradiation, which was changed to $420 \mathrm{~nm}$. Samples for fluorescence microscopy were prepared according to a previous report. ${ }^{37}$ Fluorescence derived from GaTPP was observed on a fluorescence microscope (DMI4000B, Leica) equipped with a Y3 filter cube (Excitation filter: 530-560 nm, Suppression filter: $572.5-647.5 \mathrm{~nm}$ ).

\section{Data availability}

The refined models and corresponding structure factors are deposited under Protein Data Bank (PDB) accession codes 7EMO (FeTPP-HasA), 7EMP (CrTPP-HasA), 7EMQ (MnTPP-HasA), 7EMR (CoTPP-HasA), 7EMS (L85A mutant of FeTPP-HasA), and 7VM1 (FeTPyP-HasA).

\section{References}

1. Groves, J.T. \& Watanabe, Y. Reactive iron porphyrin derivatives related to the catalytic cycles of cytochrome P-450 and peroxidase. Studies of the mechanism of oxygen activation. J. Am. Chem. Soc. 110, 8443-8452 (1988).

2. Collman, J.P., Chien, A.S., Eberspacher, T.A. \& Brauman, J.I. Multiple Active Oxidants in Cytochrome P-450 Model Oxidations. J. Am. Chem. Soc. 122, 11098-11100 (2000).

3. Suzuki, N., Higuchi, T. \& Nagano, T. Multiple active intermediates in oxidation reaction catalyzed by synthetic heme-thiolate complex relevant to cytochrome p450. J. Am. Chem. Soc. 124, 9622-9628 (2002).

4. Rothemund, P. FORMATION OF PORPHYRINS FROM PYRROLE AND ALDEHYDES. $J$. Am. Chem. Soc. 57, 2010-2011 (1935).

5. Rothemund, P. A New Porphyrin Synthesis. The Synthesis of Porphin1. J. Am. Chem. Soc. 58, 625-627 (1936).

6. Adler, A.D., Longo, F.R. \& Shergalis, W. Mechanistic Investigations of Porphyrin Syntheses. I. Preliminary Studies on ms-Tetraphenylporphin. J. Am. Chem. Soc. 86, 3145-3149 (1964).

7. $\quad$ Adler, A.D., et al. A simplified synthesis for meso-tetraphenylporphine. J. Org. Chem. 32, 476476 (1967).

8. Lindsey, J.S., Hsu, H.C. \& Schreiman, I.C. Synthesis of tetraphenylporphyrins under very mild conditions. Tetrahedron Lett. 27, 4969-4970 (1986).

9. Lindsey, J.S., Schreiman, I.C., Hsu, H.C., Kearney, P.C. \& Marguerettaz, A.M. Rothemund and Adler-Longo reactions revisited: synthesis of tetraphenylporphyrins under equilibrium conditions. J. Org. Chem. 52, 827-836 (1987).

10. Laha, J.K., Dhanalekshmi, S., Taniguchi, M., Ambroise, A. \& Lindsey, J.S. A Scalable Synthesis of Meso-Substituted Dipyrromethanes. Org. Process Res. Dev. 7, 799-812 (2003).

11. Galardon, E., Le Maux, P. \& Simonneaux, G. Cyclopropanation of Alkenes, N-H and S-H 
Insertion of Ethyl Diazoacetate Catalysed by Ruthenium Porphyrin Complexes. Tetrahedron 56, 615-621 (2000).

12. Baumann, L.K., Mbuvi, H.M., Du, G. \& Woo, L.K. Iron Porphyrin Catalyzed N-H Insertion Reactions with Ethyl Diazoacetate. Organometallics 26, 3995-4002 (2007).

13. Maid, H., et al. Iron Catalysis for In Situ Regeneration of Oxidized Cofactors by Activation and Reduction of Molecular Oxygen: A Synthetic Metalloporphyrin as a Biomimetic NAD(P)H Oxidase. Angew. Chem. Int. Ed. 50, 2397-2400 (2011).

14. Rao, H., Schmidt, L.C., Bonin, J. \& Robert, M. Visible-light-driven methane formation from CO2 with a molecular iron catalyst. Nature 548, 74-77 (2017).

15. Sudoh, K., et al. Syntheses, Properties, and Catalytic Activities of Metal(II) Complexes and Free Bases of Redox-Switchable $20 \pi, 19 \pi$, and $18 \pi$ 5,10,15,20-Tetraaryl-5,15diazaporphyrinoids. Chem. Eur. J. 23, 16364-16373 (2017).

16. Maaskant, R.V., Polanco, E.A., van Lier, R.C.W. \& Roelfes, G. Cationic iron porphyrins with sodium dodecyl sulphate for micellar catalysis of cyclopropanation reactions. Org. Biomol. Chem. 18, 638-641 (2020).

17. Berenbaum, M.C., et al. Meso-Tetra(hydroxyphenyl)porphyrins, a new class of potent tumour photosensitisers with favourable selectivity. Br. J. Cancer 54, 717-725 (1986).

18. James, D.A., Arnold, D.P. \& Parsons, P.G. POTENCY AND SELECTIVE TOXICITY OF TETRA(HYDROXYPHENYL)- AND TETRAKIS(DIHYDROXYPHENYL)PORPHYRINS IN HUMAN MELANOMA CELLS, WITH AND WITHOUT EXPOSURE TO RED LIGHT. Photochem. Photobiol. 59, 441-447 (1994).

19. Villanueva, A., Caggiari, L., Jori, G. \& Milanesi, C. Morphological aspects of an experimental tumour photosensitized with a meso-substituted cationic porphyrin. J. Photochem. Photobiol. B Biol. 23, 49-56 (1994).

20. Abrahams, B.F., Hoskins, B.F., Michail, D.M. \& Robson, R. Assembly of porphyrin building blocks into network structures with large channels. Nature 369, 727-729 (1994).

21. Hagrman, D., Hagrman, P.J. \& Zubieta, J. Solid-State Coordination Chemistry: The SelfAssembly of Microporous Organic-Inorganic Hybrid Frameworks Constructed from Tetrapyridylporphyrin and Bimetallic Oxide Chains or Oxide Clusters. Angew. Chem. Int. Ed. 38, 3165-3168 (1999).

22. Sharma, C.V.K., et al. Design Strategies for Solid-State Supramolecular Arrays Containing Both Mixed-Metalated and Freebase Porphyrins. J. Am. Chem. Soc. 121, 1137-1144 (1999).

23. Sharma, C.V.K., Broker, G.A. \& Rogers, R.D. Polymorphous One-Dimensional Tetrapyridylporphyrin Coordination Polymers Which Structurally Mimic Aryl Stacking Interactions. J. Solid State Chem. 152, 253-260 (2000).

24. Beyzavi, M.H., et al. A Hafnium-Based Metal-Organic Framework as a Nature-Inspired Tandem Reaction Catalyst. J. Am. Chem. Soc. 137, 13624-13631 (2015).

25. Rickhaus, M., et al. Global aromaticity at the nanoscale. Nat. Chem. 12, 236-241 (2020). 
26. Key, H.M., Dydio, P., Clark, D.S. \& Hartwig, J.F. Abiological catalysis by artificial haem proteins containing noble metals in place of iron. Nature 534, 534-537 (2016).

27. Sreenilayam, G., Moore, E.J., Steck, V. \& Fasan, R. Metal Substitution Modulates the Reactivity and Extends the Reaction Scope of Myoglobin Carbene Transfer Catalysts. Adv. Synth. Catal. 359, 2076-2089 (2017).

28. Omura, K., et al. Reconstitution of full-length P450BM3 with an artificial metal complex by utilising the transpeptidase Sortase A. Chem. Commun. 54, 7892-7895 (2018).

29. Oohora, K., Miyazaki, Y. \& Hayashi, T. Myoglobin Reconstituted with Ni Tetradehydrocorrin as a Methane-Generating Model of Methyl-coenzyme M Reductase. Angew. Chem. Int. Ed. 58, 13813-13817 (2019).

30. Hirayama, S., Oohora, K., Uchihashi, T. \& Hayashi, T. Thermoresponsive Micellar Assembly Constructed from a Hexameric Hemoprotein Modified with Poly(N-isopropylacrylamide) toward an Artificial Light-Harvesting System. J. Am. Chem. Soc. 142, 1822-1831 (2020).

31. Bender, G.M., et al. De Novo Design of a Single-Chain Diphenylporphyrin Metalloprotein. $J$. Am. Chem. Soc. 129, 10732-10740 (2007).

32. Polizzi, N.F., et al. De novo design of a hyperstable non-natural protein-ligand complex with sub-Å accuracy. Nat. Chem. 9, 1157-1164 (2017).

33. Pirro, F., et al. Allosteric cooperation in a de novo-designed two-domain protein. Proc. Natl. Acad. Sci. 117, 33246 (2020).

34. Shirataki, C., et al. Inhibition of Heme Uptake in Pseudomonas aeruginosa by its Hemophore (HasA p ) Bound to Synthetic Metal Complexes. Angew. Chem. Int. Ed. 53, 2862-2866 (2014).

35. Uehara, H., et al. Structures of the Heme Acquisition Protein HasA with Iron(III)-5,15Diphenylporphyrin and Derivatives Thereof as an Artificial Prosthetic Group. Angew. Chem. Int. Ed. 56, 15279-15283 (2017).

36. Sakakibara, E., et al. Highly malleable haem-binding site of the haemoprotein HasA permits stable accommodation of bulky tetraphenylporphycenes. RSC Advances 9, 18697-18702 (2019).

37. Shisaka, Y., et al. Hijacking the Heme Acquisition System of Pseudomonas aeruginosa for the Delivery of Phthalocyanine as an Antimicrobial. ACS Chem. Biol. 14, 1637-1642 (2019).

38. Tacconelli, E., et al. Discovery, research, and development of new antibiotics: the WHO priority list of antibiotic-resistant bacteria and tuberculosis. Lancet Infect. Dis. 18, 318-327 (2018).

39. Bondi, A. van der Waals Volumes and Radii. The Journal of Physical Chemistry 68, 441-451 (1964).

40. Kataoka, H., et al. Analysis of the influence of drug resistance factors on the efficacy of combinations of antibiotics for multidrug-resistant Pseudomonas aeruginosa isolated from hospitals located in the suburbs of Kanto area, Japan. Journal of Global Antimicrobial Resistance 1, 91-96 (2013).

41. Lin, Q., et al. Porphyrinic coordination lattices with fluoropillars. Journal of Materials 
Chemistry A 5, 21189-21195 (2017).

42. Bizaia, N., et al. Porphyrin-kaolinite as efficient catalyst for oxidation reactions. ACS Appl. Mater. Interfaces 1, 2667-2678 (2009).

43. Das, S.K., et al. Electron Transfer Studies of High Potential Zinc Porphyrin-Fullerene Supramolecular Dyads. J. Phys. Chem. C 118, 3994-4006 (2014).

44. Yukl, E.T., et al. Kinetic and Spectroscopic Studies of Hemin Acquisition in the Hemophore HasAp from Pseudomonas aeruginosa. Biochemistry 49, 6646-6654 (2010).

45. Kabsch, W. XDS. Acta Crystallogr., Sect. D: Biol. Crystallogr. 66, 125-132 (2010).

46. Yamashita, K., Hirata, K. \& Yamamoto, M. KAMO: towards automated data processing for microcrystals. Acta Crystallogr. D Struct. Biol. 74, 441-449 (2018).

47. Otwinowski, Z. \& Minor, W. Processing of X-ray diffraction data collected in oscillation mode. in Methods Enzymol., Vol. 276 307-326 (Academic Press, 1997).

48. Evans, P. Scaling and assessment of data quality. Acta Crystallogr., Sect. D: Biol. Crystallogr. 62, 72-82 (2006).

49. Evans, P.R. An introduction to data reduction: space-group determination, scaling and intensity statistics. Acta Crystallogr., Sect. D: Biol. Crystallogr. 67, 282-292 (2011).

50. Evans, P.R. \& Murshudov, G.N. How good are my data and what is the resolution? Acta Crystallogr., Sect. D: Biol. Crystallogr. 69, 1204-1214 (2013).

51. Vagin, A. \& Teplyakov, A. MOLREP: an Automated Program for Molecular Replacement. $J$. Appl. Crystallogr. 30, 1022-1025 (1997).

52. Alontaga, A.Y., et al. Structural Characterization of the Hemophore HasAp from Pseudomonas aeruginosa: NMR Spectroscopy Reveals Protein - Protein Interactions between Holo-HasAp and Hemoglobin. Biochemistry 48, 96-109 (2009).

53. Emsley, P. \& Cowtan, K. Coot: model-building tools for molecular graphics. Acta Crystallogr., Sect. D: Biol. Crystallogr. 60, 2126-2132 (2004).

54. Murshudov, G.N., Vagin, A.A. \& Dodson, E.J. Refinement of macromolecular structures by the maximum-likelihood method. Acta Crystallogr., Sect. D: Biol. Crystallogr. 53, 240-255 (1997).

55. Murshudov, G.N., et al. REFMAC5 for the refinement of macromolecular crystal structures. Acta Crystallogr., Sect. D: Biol. Crystallogr. 67, 355-367 (2011).

56. Kovalevskiy, O., Nicholls, R.A., Long, F., Carlon, A. \& Murshudov, G.N. Overview of refinement procedures within REFMAC5: utilizing data from different sources. Acta Crystallogr. D Struct. Biol. 74, 215-227 (2018).

57. Schuttelkopf, A.W. \& van Aalten, D.M. PRODRG: a tool for high-throughput crystallography of protein-ligand complexes. Acta Crystallogr., Sect. D: Biol. Crystallogr. 60, 1355-1363 (2004).

58. Moriarty, N.W., Grosse-Kunstleve, R.W. \& Adams, P.D. electronic Ligand Builder and Optimization Workbench (eLBOW): a tool for ligand coordinate and restraint generation. Acta Crystallogr., Sect. D: Biol. Crystallogr. 65, 1074-1080 (2009). 
59. Moore, E.J., Zorine, D., Hansen, W.A., Khare, S.D. \& Fasan, R. Enzyme stabilization via computationally guided protein stapling. Proc. Natl. Acad. Sci. 114, 12472 (2017).

60. Ravel, B. \& Newville, M. ATHENA, ARTEMIS, HEPHAESTUS: data analysis for X-ray absorption spectroscopy using IFEFFIT. J. Synchrotron Radiat. 12, 537-541 (2005).

\section{Acknowledgements}

This work was supported by JSPS KAKENHI Grant Number JP15H05806 and JP18H02084 to O.S., JP18J15250 to Y.S., and JP18J23340 to K.S. This work was also supported by JST CREST Grant Number JPMJCR15P3 to O.S. and the SUNBOR scholarship from the SUNTORY FOUNDATION for LIFE SCIENCES to Y.S. The authors thank the staff at RIKEN SPring-8 centre BL26B1, BL26B2, and BL41XU for their technical support with data collection (proposal numbers 2018A2576, 2019A2519, 2020A2556, 20180028, 20190050, 20200045). We would like to extend our gratitude to Prof. Mizuki Tada and Asst. Prof. Hirosuke Matsui for their assistance with XANES measurements.

\section{Author contributions}

Y.S. and O.S. conceived the project. Y.S., E.S., H.O., G.U., and H.S. performed protein purification and crystallographic analysis. E.S. measured CD spectra. H.O. performed XANES analysis. E.S. carried out growth inhibition and photosterilisation experiments with the help of M.H. Y.S. wrote the first draft of the manuscript. Y.S., J.K.S., K.S., and O.S. prepared the manuscript. All authors discussed the manuscript and approved the final version.

\section{Competing interests}

The authors declare no competing interests.

\section{Additional information}

Supplementary information The online version contains supplementary material available.

Correspondence and requests for materials should be addressed to O.S. 